Pesq. Vet. Bras. 36(12):1221-1226, dezembro 2016 DOI: $10.1590 / \mathrm{S} 0100-736 \mathrm{X} 2016001200014$

\title{
Quantification of collagen fibers in canine uteri treated with medroxyprogesterone acetate ${ }^{1}$
}

\author{
Paulo Salinas ${ }^{2,3,4}$, Maria A. Miglino ${ }^{5}$ and Mariano del Sol ${ }^{6,7}$
}

\begin{abstract}
Salinas P., Miglino M.A. \& del Sol M. 2016. Quantification of collagen fibers in canine uteri treated with medroxyprogesterone acetate. Pesquisa Veterinária Brasileira 36(12):1221-1226. Department of Basic Sciences, Faculty of Medicine, University of La Frontera, Campus Andrés Bello, Montevideo s/n, Temuco, Chile. E-mail: mariano.delsol@ufrontera.cl

Collagen plays essential roles in remodeling uterine tissue during decidualization, implantation, pregnancy and involution. To understand whether the progestational agent medroxyprogesterone acetate (MPA) can modify the organization and deposit of collagen in the uteri of normal bitches (Canis Tlupus familiaris), we assessed uterine tissues by histochemistry. Uteri were grouped as: nulliparous $(\mathrm{n}=11)$, multiparous $(\mathrm{n}=11)$ and treated with MPA ( $\mathrm{n}=11$; nulliparous with two treatments; $5 \mathrm{mg} / \mathrm{kg}$; i.m.). The amount, location and birefringence of interstitial collagen types I and III in the fold and base of the endometrial stroma and the myometrial muscular layers were studied on sections stained with Picrosirius Red by polarized light microscopy and evaluated by ANOVA. No differences were observed in the endometrium. In the myometrium, differences were observed in collagen type I between MPA-treated and nulliparous uteri vs. multiparous $(p<0.05)$, and differences in collagen type III between nulliparous and multiparous uteri vs. MPA-treated $(p=0.0001)$. In conclusion, two doses of MPA had no significant effect on the investigated collagens in the extracellular matrix.
\end{abstract}

INDEX TERMS: Canis lupus familiaris, uterus, medroxyprogesterone acetate, collagen, Picrosirius red.

\section{INTRODUCTION}

Collagen in uterus is the most abundant protein of the extracellular matrix (ECM) and the most important structural component of the connective tissue. It is the molecular scaffolding, providing strength and lending support to the endometrial and myometrial tissue; it is also involved in tissue processes such as growth, differentiation, repair and other pathological conditions, as well as in reproductive

\footnotetext{
${ }^{1}$ Received on April 23, 2016.

Accepted for publication on August 11, 2016.

${ }^{2}$ Institute of Biology, Pontificia Universidad Católica de Valparaíso, Campus Curauma, Valparaíso, Chile.

${ }^{3}$ Laboratory of Veterinary Anatomy, School of Veterinary Medicine, Faculty of Veterinary Medicine and Natural Resources, Universidad Santo Tomás, Manuel Rodríguez 060, Temuco, Chile.

${ }^{4} \mathrm{PhD}$ Program in Morphological Sciences, Faculty of Medicine, Universidad de La Frontera, Campus Andrés Bello, Montevideo s/n, Temuco, Chile.

${ }^{5}$ Department of Surgery, Faculty of Veterinary Medicine and Animal Science, University of São Paulo (USP), Av. Prof. Dr. Orlando Marques de Paiva 87, Cidade Universitária, São Paulo, SP 05508-270, Brazil.

${ }^{6}$ Department of Basic Sciences, Faculty of Medicine, Universidad de La Frontera, Campus Andrés Bello, Montevideo s/n, Temuco, Chile.

${ }^{7}$ Center for Research in Biomedical Sciences, Universidad Autónoma de Chile, Chile. *Corresponding author: mariano.delsol@ufrontera.cl
}

processes such as decidualization, implantation, pregnancy and subsequent involution. Collagen type I provides rigidity and is frequently described as being co-located with collagen type III, which provides elasticity.

In most species, and depending on their reproductive state, adaptive phenotypic changes occur in the uterus, modulated by hormonal variations and mechanical influences that modify its shape (size and weight) and biomechanical properties (distension and growth). At the tissue level, cellular hyperplasia and hypertrophy of the muscular tissue and increased synthesis and deposit of ECM protein (collagen, elastin and adhesion proteins) are observed. Studies on rats have described the molecular mechanism that controls ECM protein gene expression and collagen synthesis. The evidence suggests that in a female with a normal cycle and under the influence of estrogen the myometrium smooth muscle cells (MSMC) can synthesize collagen. Conversely, during late pregnancy there is a significant increase in the amount of collagen types I and III, which suggests that this condition has a major role in inducing changes in the biochemical properties of the MSMC in response to the influence of progesterone $\left(\mathrm{P}_{4}\right)$ to express ECM protein genes and synthesize collagen (Ross \& Klebanoff 
1971, Stewart et al. 1995). Reports have shown a decrease in collagen expression and a coordinated temporal increase in the expression of components of the basement membrane near term associated with decreased $\mathrm{P}_{4}$ (Shynlova et al. 2004).

The ovary mainly synthesizes $\mathrm{P}_{4}$ and it is stimulated by the pulsatile release of adenohypophyseal luteinizing hormone (LH). This is crucial during the estrous cycle and during late bitch pregnancy. It has been shown that $\mathrm{P}_{4}$ delays uterine involution postpartum and collagen uterine loss in rats and other mammals (Halme \& Woessner 1975). Progestins are synthetic steroidal hormones with progestational activity and progesterone derivatives. They are widely used in bitches, with indications that range from antineoplastic treatments to dermatological problems; however, their main use has been control of the reproductive cycle. One of the most frequently used in clinical veterinary medicine is medroxyprogesterone acetate (MPA), which affects collagen synthesis by inhibiting the expression of extracellular matrix metalloproteinases (MMPs) induced by pro-inflammatory cytokines, such as tumor necrosis factor alpha $(\mathrm{TNF} \alpha)$ in deciduous cells and the uterine stroma (Oner et al. 2008, Schatz et al. 2006, Strakova et al. 2003). MMPs are proteins with enzymatic properties capable of decomposing ECM collagen; they participate in wound repair and tissue remodeling. Therefore, MMPs and their natural or synthetic tissue inhibiters are molecules that regulate myometrial growth and remodeling as well as collagen distribution. Another effect of MPA is said to be the increase in the baseline concentration of gonadotropins, particularly in the pulses of adenohypophyseal pulsatile LH from the main endocrine promoter of the ovarian $\mathrm{P}_{4}$ synthesis (Beijerink et al. 2008). Moreover, it has been reported that MPA induces the secretion of insulin-like growth factor (IGF-I; Beijerink et al. 2007), which has been shown to be a powerful stimulator of protein synthesis and collagen gene expression (Gillery et al. 1992).

Despite the background regarding the effects of MPA in primates and rats on the mechanisms that activate or inhibit collagen synthesis, there is little evidence of its effects on the deposit, amount and location of interstitial collagen when used as a contraceptive in bitches during the anestrus cycle and how these effects could influence such biological processes as implantation, gestation and subsequent involution. The aim of the study was to quantify the histochemistry expression of collagen types I and III in the canine uterus to determine the effect of MPA when used as a contraceptive.

\section{MATERIALS AND METHODS}

Ethics statement. This study was conducted at the Center for Excellence in Surgical and Morphological Studies of the Universidad de La Frontera, Temuco, Chile. The animals were handled in accordance with guidelines for animal research as detailed in the NIH Guidelines for the Care and Use of Laboratory Animals (NIH 1985 ) with ethical approval from the Universidad Santo Tomás Research Ethics Committee CE 081/2013.

Animals, samples and determination of estrous cycle. Uterine horn samples were obtained from 33 healthy adult bitches (2-6 years; Canis lupus familiaris) during the anestrous cycle, of no defined breed, by means of ovariohysterectomy (OVH). A vaginal inspection was performed as well as a cytological evaluation of the vaginal floor. Three groups were formed: Nulliparous $(n=11$; older than 2 years and that has had at least one estrous cycle), Multiparous ( $\mathrm{n}=11$; older than 2 years and that had carried at least two gestations to term) and MPA-treated ( $n=11$; older than 2 years, nulliparous that been had exposed twice to contraceptive treatment during the anestrous cycle). The treatment was done with the progestin OVO- $6^{\circledR} 50 \mathrm{mg}$ (Laboratorio Drag Pharma Chile Invetec S.A.) by intramuscular injection applied twice at an 8-week interval $(5 \mathrm{mg} / \mathrm{kg})$. ). The anestrus was evaluated using physical examination, vaginal cytology (Diff Quick ${ }^{\circledR}$, Hartman Leddon Co.; Thrall \& Olson 1999), histological parameters of the uterus and ovaries (Rehm et al. 2007, Concannon 2011) and confirmed by the absence of the corpus luteum in the ovary.

Preparation of the uterine tissue. Sections of uterine horn were obtained and fixed in $10 \%$ formaldehyde for $72 \mathrm{~h}$ at room temperature $\left(22^{\circ} \mathrm{C}\right)$. Then, they were dehydrated in a series of alcohols, clarified in xylol and embedded in Paraplast (Paraplast Plus embedding medium; melting point: $54^{\circ} \mathrm{C}$; Sigma-Aldrich Chemical Co., St Louis, MO, USA). Serial sections were made, $5 \mu \mathrm{m}$ thick, with a motorized rotary microtome Leica RM2255 (Leica Microsystems, Switzerland). Then, the sections were rehydrated, immersed in xylol (10 min.), exposed to descending concentrations of ethanol $(100 \%, 96 \%, 80 \%$ and $70 \%$, all $15 \mathrm{~s})$ and finally distilled water. Staining was performed using $0.1 \%$ solution of Picrosirius red in a saturated aqueous solution of picric acid for one hour at room temperature (Junqueira et al. 1979). Then, sections were rinsed in $0.01 \mathrm{NHCl}$ for $2 \mathrm{~min}$, dehydrated in ascending concentrations of ethanol $(70 \%, 80 \%, 96 \%$ and $100 \%$, each 10 sec) and cleared in two stages in xylene, 10 min each. Sections were covered with Entellan mounting medium (Merck, Darmsta$\mathrm{dt}$, Germany) and a glass cover slip. Picrosirius red staining is a frequently used histochemical selective technique for the detection of collagen in paraffin-embedded histological sections. The collagen present in the ECM displays different colors from interference and birefringence spectra; therefore, it is useful for the differential study of the distribution of different types of collagen (Borges et al. 2007).

Determination of collagen. The uterus sections were observed by polarization microscopy using a Leica ${ }^{\circledR}$ DM750 optical microscope (Leica Microsystems, Switzerland) equipped with a light circular polarizer composed of a simple polarizer and a filter analyzer (Leica Microsystems, Switzerland). This filter was aligned so the background in the field of view would be as dark as possible. Collagen type III (fibrillar-immature) was identified by green birefringence, whereas collagen type I (dense-mature) was identified by yellow-red birefringence (Junqueira et al. 1979). Digital images (40X) of uterine layers were obtained with a Leica ${ }^{\circledR}$ MC170HD digital camera using Leica Acquire v3.2 software for Mac OS X (Leica Microsystems, Switzerland). Five random fields were evaluated in each uterine layer (endometrium: fold and base; myometrium: circular and longitudinal muscle layer). Two scans were made from each field, one with normal light and another with polarized light. Image analysis was done using ImageJ v1.49 software (National Institute of Health, USA). The collagen type I and III content was calculated using the software's "threshold" function and "color segmentation" plugin (EPFL, Lausanne, Switzerland) and was expressed as a fraction area (\%) of each image (Schindelin 2015). This ImageJ plugin allows a color image or a stack of color to be segmented by pixel clustering. The cluster was defined manually by the user through the interface. 220 images (2048 x1536 pixels) were analyzed per group.

Statistical analyses. Data were expressed as mean \pm SD. The D’Agostino-Pearson test was used to detect data normality. Data for 
the fraction area of collagen type I and III on the fold and base of the endometrium, circular muscle layer and longitudinal muscle layer were analyzed by a one-way ANOVA among the three groups and Tukey's post-test of multiple comparisons was used. Statistical significance for all tests of hypotheses was established as $p<0.05$ (Zar 1999). The data analysis was performed using GraphPad Prism v. 5.0 software for Mac OS X (GraphPad Software, San Diego CA).

\section{RESULTS}

\section{Histology}

Nulliparous: Longitudinal folds covered by a columnar simple epithelium were observed in the endometrium. The endometrial lamina propria presented lax collagenous connective tissue and tubular glands covered by a cuboidal simple epithelium. The circular and longitudinal muscle layers of the myometrium appeared well-defined, surrounded by lax collagenous connective tissue and covered by a thin serous membrane. Under polarized light, a red-orange and green birefringence spectrum was observed, suggesting the presence of collagen types I and III, respectively, distributed homogenously in the endometrium and myometrium. Type I and III collagen fibers were observed, forming a fine mesh homogenous in length and diameter around the endometrial glands; however, a small amount was detected under the epithelial lining. In the basal region of the endometrial mucosa, a more intense green birefringence was noted, suggesting the presence type III collagenous fibers grouped in organized interwoven bundles around the glandular epithelium. In blood vessels of the vascular layer of the myometrium, collagen type I was identified in the tunica externa and type III in the tunica externa, media and intima. Multiparous: Irregular folds covered by a columnar simple epithelium were observed in the endometrium. The endometrial lamina propria presented lax collagenous connective tissue and glands surrounded by collagenous fibers forming a mesh. The circular muscle layer was well-defined. The longitudinal muscle layer had muscle fibers with heterogeneous direction and lax collagenous connective tissue. Under polarized light, a red-orange and green birefringence spectrum was observed in the endometrium and myometrium suggesting the presence of collagen types I and III, respectively, distributed homogenously. In the basal region of the endometrial mucosa a larger number of type III collagenous fibers was observed, grouped in interwoven bundles around the glandular epithelium. The circular muscle layer had a predominance of collagen type III in an organized arrangement. In blood vessels of the vascular layer of the myometrium, collagen type I was identified in the tunica intima and externa, and a greater birefringence spectrum of collagen type III in the tunica externa, media and intima. Treated with MPA: Irregular folds were observed in the endometrium, covered by cuboidal simple epithelium and lax collagenous connective tissue in the lamina propria and irregular glands covered by cuboidal simple epithelium. Endometrial glands surrounded by collagenous fibers of a homogenous diameter were noted, as well as fibers smaller in diameter distributed with no apparent order. The circular muscle layer exhibited small cells. Less thick oblique and longitudinal cells were observed in the longitudinal muscle layer. Under polarized light, a red-orange and green birefringence spectrum was observed in the endometrium and myometrium suggesting the presence of collagen types I and III, respectively, distributed homogenously. The circular muscle layer had a predominance of collagen type III; in addition, a greater birefringence compared to the other groups was observed. In blood vessels of the vascular layer of the myometrium, collagen type I was identified in the tunica externa and media, with a greater birefringence spectrum of collagen type III in the tunica externa, media and intima (Fig.1).

\section{Quantification of collagen}

The analysis of uterine wall sections stained with Picrosirius red and observed via polarization microscopy revealed that in the endometrium, generally, no differences were detected in the amount of collagen types I and III (area fraction; \%) between groups. In the myometrium, in terms of collagen type I (Table 1), differences were noted between MPA-treated and Nulliparous vs. Multiparous uteri $(p<0.05)$, and in collagen type III differences were observed between Nulliparous and Multiparous vs. MPA-treated $(p=0.0001)$. Generally, the evidence obtained in this study regarding collagen types I and III did not make it possible to detect differences between MPA-treated and nulliparous uteri. A smaller amount of collagen was found in the Multiparous group compared to the other study groups, mainly in the circular $(p=0.0001)$ and longitudinal muscle layers $(p=0.0003)$. In addition, the study revealed a significant increase in the number of type III collagenous fibers in the longitudinal muscle layer of MPA-treated bitches $(14.98 \pm 5.05)$, detecting differences $(p=0.0001)$ to uteri from nulliparous $(11.05 \pm 3.41)$ and multiparous bitches (8.55 \pm 2.37 ; Table 2).

\section{DISCUSSION}

The use of the synthetic progestin MPA is frequent in clinical veterinary medicine and together with megestrol acetate, proligestone and gonadotropin-releasing hormone $(\mathrm{GnRH})$, these are the most frequently used drugs for nonsurgical contraception in bitches and cats. MPA is widely used due to its low cost and ease of acquisition among veterinarians and non-veterinarians. Medical indications include endocrinal disorders, behavioral treatment and neoplasia control; however, its main use is population control due to its contraceptive effect, blocking the synthesis and release of hypothalamic GnRH. Yet despite the usefulness of its therapeutic effects, numerous side effects have been described (Monteiro et al. 2009). Reports indicate that the clinical and/or endocrinal effect on the reproductive system varies according to the dose and time of application with respect to the estrous cycle.

The proteins that constitute the ECM have a dominant role in the structure and function of the tissues and organs. The amount, diameter and distribution of collagen present in the uterus will directly influence the tensile strength and elastic properties of the tissue (Ottani et al. 2001). It is known that interstitial collagen is not homogenous, but it is composed mainly of collagen types I and III. Studies that analyze and quantify the possible functional significance of 
the degree of heterogeneity of both collagen types in normal or pathological tissue have shown that tissue structure and function are associated with the relative amounts and proportions of ECM proteins, mainly of the collagen, and corroborate the physiological importance of the endometrial stroma and its proteins (Hance \& Crystal 1977, Boos 2000). In the present study, we analyzed the effect of the MPA administration on deposit of uterine collagen in the ECM and demonstrated the coexistence of collagen type I and type III in canine uterus.

We have observed a predominance of collagen type III, its expression being particularly evident in all strata of the uteri of MPA-treated, nulliparous and multiparous bitches studied. From the qualitative observation there are no significant differences between the three study groups in the distribution and orientation of collagen types I and III. The quantitative evidence demonstrated a ratio between collagen types I and III in the uteri of nulliparous, multiparous and MPA-treated bitches of 1:3,1:4 and 1:3.5, respectively. These data agree with those reported by Augsburger \& Henzi (2008) in uteri of nulliparous and multiparous canine females, who demonstrated an identical immunoreactivity in collagen types I and III using immunohistochemical techniques; nevertheless, the intensity of immunostaining observed in collagen
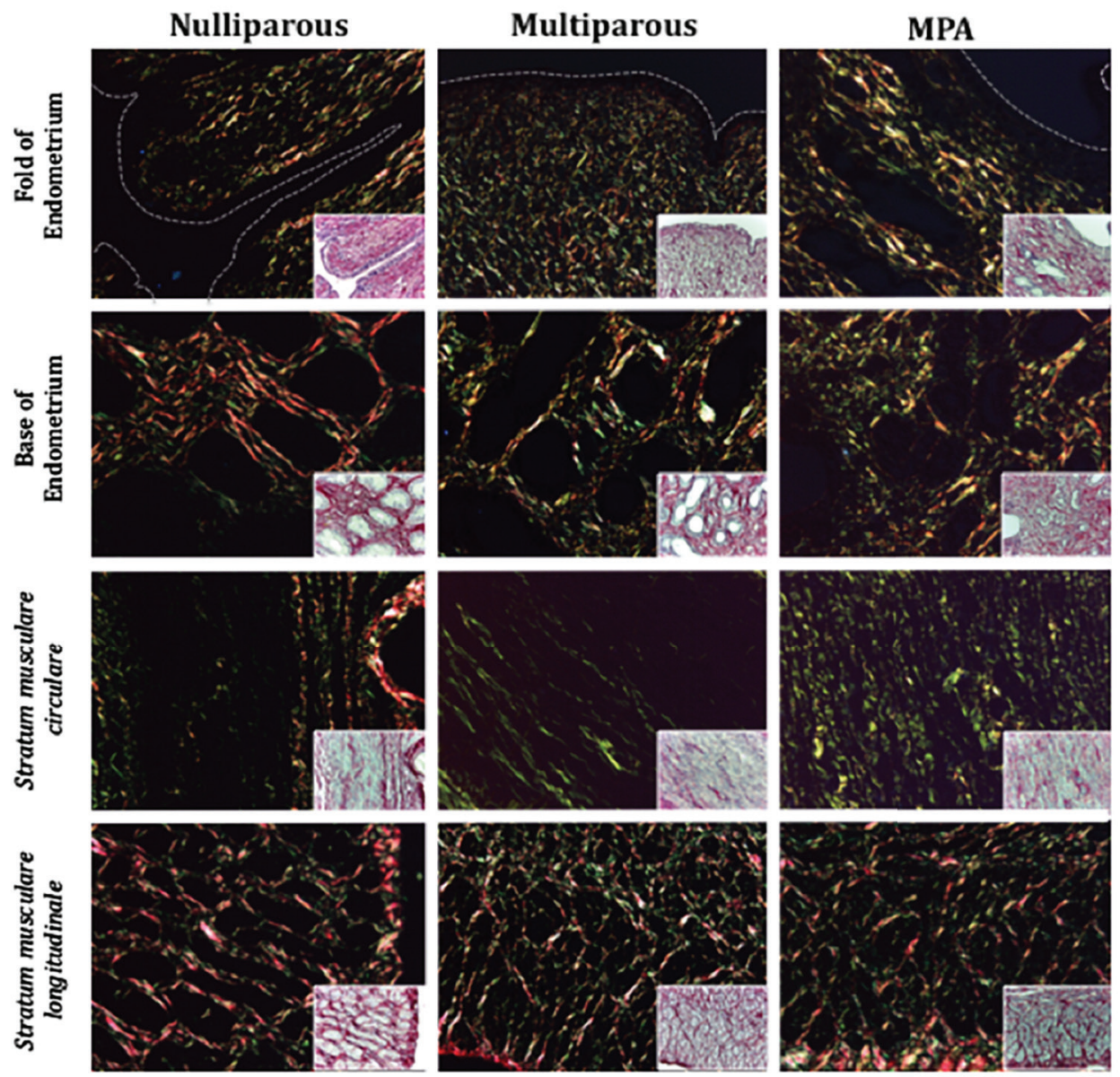

Fig.1. Picrosirius stained sections from the canine uterine horn. Obj.40x.

Table 1. Quantification (fraction area; \%) of collagen type I on the uterine wall (n=11)

\begin{tabular}{lccccc}
\hline & & Nulliparous & Multiparous & MPA-treated & P value \\
\hline \multirow{2}{*}{ Endometrium } & Endometrial fold & $2.076 \pm 0.798$ & $1.914 \pm 1.096$ & $1.936 \pm 1155$ & 0.8642 \\
& Lamina propria & $4.014 \pm 2.168$ & $3.375 \pm 1.821$ & $2.843 \pm 1.555$ & 0.2620 \\
\multirow{2}{*}{ Myometrium } & Circular muscle layer & $3.499 \pm 2.418^{\mathrm{a}}$ & $1.579 \pm 1.305^{\mathrm{b}}$ & $4.127 \pm 1.213^{\mathrm{a}}$ & 0.0001 \\
& Longitudinal muscle layer & $4.621 \pm 1.801^{\mathrm{a}}$ & $3.155 \pm 1.395^{\mathrm{b}}$ & $6.871 \pm 4.628^{\mathrm{a}}$ & 0.0003
\end{tabular}

Table 2. Quantification (fraction area; \%) of collagen type III on the uterine wall (n=11)

\begin{tabular}{lccccc}
\hline & & Nulliparous & Multiparous & MPA-treated & p-value \\
\hline \multirow{2}{*}{ Endometrium } & Endometrial fold & $9.848 \pm 4.178$ & $9.748 \pm 4.509$ & $9.199 \pm 4.604$ & 0.9001 \\
& Lamina propria & $10.75 \pm 3.458$ & $13.64 \pm 8.136$ & $14.97 \pm 6.918$ & 0.0630 \\
\multirow{2}{*}{ Myometrium } & Circular muscle layer & $12.68 \pm 4.925$ & $9.470 \pm 8.202$ & $15.25 \pm 8.061$ & 0.0224 \\
& Longitudinal muscle layer & $11.05 \pm 3.411^{\text {a }}$ & $8.559 \pm 2.377^{\text {a }}$ & $14.98 \pm 5.052^{\mathrm{b}}$ & 0.0001
\end{tabular}


type III was greater than type I in both study groups. It is worth noting that the uteri of MPA-treated bitches generally presented a greater amount of collagen in each of the layers evaluated compared to the other study groups. However, our data contrast with those obtained in rodents by Junqueira et al. (1978) and Shynlova et al. (2004), who reported that collagen type I was predominant in the uterus, possibly reaching a ratio of 2:1, 3:1 and even 5:1 over collagen type III, evidence that appears largely in flexible tissues.

Shynlova et al. (2004) explained the role of progesterone in collagen synthesis in the uterus, showing a low capacity for ECM gene expression in non-pregnant uteri, which demonstrates limited properties to synthesize collagen in these uteri in the absence of luteal progesterone. In addition, they mainly attribute the increase or decrease in uterine collagen to the control that progesterone exercises during early and late pregnancy on collagenolysis through disruption or partial degradation. In our study, MPA-treated uteri did not exhibit any dramatic changes in the collagen content compared to those exposed to luteal progesterone under conditions of nulliparity and multiparity, demonstrating that MPA, although a synthetic molecule, can prevent collagen degradation in the canine uterus, suggesting exogenous progestin can attenuate genetic transcription on some level, inhibiting collagenolysis, exerting anti-collagenolytic properties typical of the physiological progesterone previously described (Halme \& Woessner 1975). Accordingly, it is possible to suggest that in the uterus, the MPA administered in a dose of $5 \mathrm{mg} / \mathrm{kg}$ would inhibit collagenase expression and would modulate ECM proteins and therefore its biomechanical properties.

In the endometrium, the evidence obtained in our study allows us to state that there are no differences between groups in terms of the amount of collagen types I and III. By contrast, in the myometrium differences were detected between nulliparous and MPA-treated vs. multiparous uteri. This suggests that there are no differences in the amount of collagen present in the uterus of a nulliparous bitch and one treated, at least on two occasions, with MPA. We believe that this is explained by the MPA-treated uteri belonging to bitches with no history of previous pregnancies. Therefore, our study confirms that the uteri of nulliparous and MPA-treated bitches do not generally differ in terms of the amount of collagen types I and III, which leads us to hypothesize that MPA would not succeed in modifying the relative amounts of collagen present in the ECM, either through the inhibition of collagenases or the modulation of the ECM protein expression.

It has been shown that pregnancy-induced mechanical distension of the uterine wall stimulates collagen synthesis through gene expression regulation (Lye 1994). However, in our study, the uteri of multiparous bitches generally displayed relatively lower amounts of collagen types I and III than the other study groups. This can be explained by factors involved in the uterine remodeling observed in successive pregnancies. There are reports on bitches exposed to endocrine treatments that simulate pregnancy and successive gestations which describe an increase in MMP-2 levels, an indicator of tissue damage during postpartum uterine remodeling and the resulting increase in collagenolytic activity
(Coughlan et al. 1998, Chu Py et al. 2002). It should be pointed out that the activity of these enzymes and their inhibiters is dependent on hormones and their synthetic analogs (Luck et al. 1995). We hypothesize that successive gestations and consequently uterine involutions could affect the synthesis of MMPs, by inhibiting the protein expression and synthesis, the activation mechanisms or the enzymatic activity.

\section{CONCLUSION}

Our data demonstrate that two doses of MPA $(5 \mathrm{mg} / \mathrm{kg}$; i.m.), in general, do not have a significant effect on the investigated collagens in the extracellular matrix.

Acknowledgements.- This research was supported by: Fondos para Proyectos Internos de Investigación \& Creación 2014/2015, Universidad Santo Tomás (Grant $\mathrm{N}^{\circ} 16047$ ) and Programa Formación de Capital Humano Avanzado, Beca Doctorado Nacional, CONICYT (Grant D-21140825).

Conflict of interest statement.- None of the authors have any conflicts of interest to declare.

Author contributions.- All the authors collaborated in the writing and bibliographic review. Paulo Salinas coordinated the work.

\section{REFERENCES}

Augsburger H.R. \& Henzi D. 2008. Immunohistochemical expression of collagen types I, III, IV and a-actin in the uterine horns of nulliparous and multiparous beagles. Theriogenology 69:1070-1076.

Beijerink N.J., Bhatti S.F., Okkens A.C., Dieleman S.J., Mol J.A., Duchateau L., Van Ham L.M. \& Kooistra H.S. 2007. Adenohypophyseal function in bitches treated with medroxyprogesterone acetate. Domest. Anim. Endocrinol. 32:63-78.

Beijerink N.J., Bhatti S.F., Okkens A.C., Dieleman S.J., Duchateau L. \& Kooistra H.S. 2008. Pulsatile plasma profiles of FSH and LH before and during medroxyprogesterone acetate treatment in the bitch. Theriogenology 70:179-185.

Borges L.F., Gutierrez P.S., Marana H.R. \& Taboga S.R. 2007. Picrosirius-polarization staining method as an efficient histopathological tool for collagenolysis detection in vesical prolapse lesions. Micron 38(6):580-583

Boos A. 2000. Immunohistochemical Assessment of Collagen Types I, III, IV and VI in Biopsy Samples of the Bovine Uterine Wall Collected during the Oestrous Cycle. Cells Tissues Organs 167:225-238.

Chu Py P.Y., Salamonsen L.A., Lee C.S. \& Wright P.J. 2002. Matrix metalloproteinases (MMPs) in the endometrium of bitches. Reproduction 123(3):467-477.

Concannon P.W. 2011. Reproductive cycles of the domestic bitch. Anim. Reprod. Sci. 124:200-210.

Coughlan A.R., Robertson D.H., Bennett D., May C., Beynon R.J. \& Carter S.D. 1998. Matrix metalloproteinases 2 and 9 in canine rheumatoid arthritis. Vet. Rec. 143:219-223.

Gillery P., Leperre A., Maquart F.X. \& Borel J.P. 1992. Insulin-like growth factor-I (IGF-I) stimulates protein synthesis and collagen gene expression in monolayer and lattice cultures of fibroblasts. J. Cell. Physiol. 152(2):389-396.

Halme J. \& Woessner J.F. 1975. Effect of progesterone on collagen breakdown and tissue collagenolytic activity in the involuting rat uterus. J. Endocrinol. 66:357-362.

Hance A.J. \& Crystal R.D. 1977. Rigid control of synthesis of collagen Types I and III by cells in culture. Nature 268:152-154.

Junqueira L.C., Cossermelli W. \& Brentani R. 1978. Differential staining of collagens type I, II and III by Sirius Red and polarization microscopy. Arch Histol. Jpn. 41(3):267-274. 
Junqueira L.C., Bignolas G. \& Brentani R.R. 1979. Picrosirius staining plus polarization microscopy, a specific method for collagen detection in tissue sections. Histochem. J. 11:447-455.

Luck M.R., Zhao Y. \& Fisher P. 1995. Intensity of expression of tissue inhibitor of metalloproteinase (TIMP-1) in ovine reproductive tissues. J. Reprod. Fertil. Abstr. Ser. 15:47.

Lye S.J. 1994. The initiation and inhibition of labor-toward a molecular understanding. Semin. Reprod. Endocrinol. 12:284-297.

Monteiro C.M.R, Perri S.H.V., de Carvalho R.G \& Koivisto M.B. 2009. Histology and morphometry in uterus horns of nulliparous bitches, multiparous ones and bitches treated with contraceptives. Pesq. Vet. Bras. 29(10):847-851.

NIH 1985. National Institutes of Health. Guide for the Care and Use of Laboratory Animals 1985. U.S. Department of Health and Human Services, Public Health Service, National Institutes of Health, Bethesda.

Oner C., Schatz F., Kizilay G., Murk W., Buchwalder L.F., Kayisli U.A., Arici A. \& Lockwood C.J. 2008. Progestin-inflammatory cytokine interactions affect matrix metalloproteinase- 1 and -3 expression in term decidual cells: implications for treatment of chorioamnionitis-induced preterm delivery. J. Clin. Endocrinol. Metabol. 93:252-259.

Ottani V., Raspanti M. \& Rugeri A. 2001. Collagen structure and functional implications. Micron 32:251-260.

Rehm S., Stanislaus D.J. \& Williams A.M. 2007. Estrous cycle-dependent histology and review of sex steroid receptor expression in dog reproductive tissues and mammary gland and associated hormone levels. Birth Defects Res. B, Dev. Reprod. Toxicol. 80:233-245.
Ross R. \& Klebanoff S.J. 1971. The smooth muscle cell. I. In vivo synthesis of connective tissue proteins. J. Cell. Biol. 50:159-171.

Schatz F., Kuczynski E., Kloosterbooer L., Krikun G., Buchwalder L.F., Rahman M. \& Lockwood C.J. 2006. Tibolone exerts progestational inhibition of matrix metalloproteinase expression in human endometrial stromal cells. Steroids 71:768-775.

Schindelin J., Rueden C.T., Hiner M.C. \& Eliceiri K.W. 2015. The ImageJ ecosystem: An open platform for biomedical image analysis. Mol. Reprod. Dev. 82(7/8):518-529.

Shynlova O., Mitchell J.A., Tsampalieros A., Langille B.L. \& Lye S.J. 2004. Progesterone and gravidity differentially regulate expression of extracellular matrix components in the pregnant rat myometrium. Biol. Reprod. 70(4):986-992.

Stewart E.A., Floor A.E., Jain P. \& Nowak R.A.1995. Increased expression of messenger RNA for collagen type I, collagen type III, and fibronectin in myometrium of pregnancy. Obstet. Gynecol. 86:417-422.

Strakova Z., Szmidt M., Srisuparp S. \& Fazleabas A.T. 2003. Inhibition of matrix metalloproteinases prevents the synthesis of insulin-like growth factor binding protein-1 during decidualization in the baboon. Endocrinology 144:5339-5346.

Thrall M.A. \& Olson P.N. 1999. The vagina, p.240-248. In: Ibid. (Eds), Diagnostic Cytology and Hematology of the Dog and Cat. 2nd ed. Mosby Inc., St Louis, Missouri.

Zar H.1999. Biostatistical Analysis. Prentice Hall, Englewood Cliffs. 718p. 